\title{
Simultaneous measurement of non-commuting observables in entangled systems
}

\author{
Erik F.G. van Heusden and Theodorus M. Nieuwenhuizen ${ }^{a}$ \\ Institute for Theoretical Physics, University of Amsterdam, Science Park 904, \\ 1090 GL Amsterdam, The Netherlands
}

Received 7 December 2018

Published online 5 March 2019

\begin{abstract}
In an EPR setup, simultaneous measurement of two noncommuting observables is considered at station $\mathcal{A}$ and, possibly after a time delay, at station $\mathcal{B}$. At each station one apparatus measures the $z$-component of a spin, while a second one measures the $x$-component of the same spin simultaneously. The dynamics of the measurements is solved explicitly. It is shown that no violations of Bell inequalities occur in Bell-like correlation experiments, and that, in fact, experiments of this type are subject to even stronger constraints. This is caused by the mutual influence of the two apparatuses coupled to the same spin, an effect which occurs at both stations.
\end{abstract}

\section{Introduction}

A measurement is a process with several possible outcomes, each of which indicates initial properties of the measured system. The effectiveness or accuracy of such a process depends on how strong the indication is. In quantum theory, it is added that, after the measurement, the measured system generally is to be found in an eigenstate of the measured observable, consistent with the pointer that is to be interpreted as the output of the measurement. Another important phenomenon in quantum mechanics is that, even in principle, sets of observable properties corresponding to non-commuting operators, that do not always have shared eigenstates, cannot be simultaneously measured, or even defined, with infinite accuracy. The indication provided by the measurement often is a statistical one at best.

Heisenberg's uncertainty principle states that this effect can not be exclusively attributed to the measurement equipment, but is a fundamental property of quantum mechanics: a system cannot be found in a state in which certain properties are defined with arbitrary precision simultaneously. If one first measures a position, and then a momentum, the execution of the second measurement invalidates the outcome of the first. One can no longer claim to know the position of the particle from the first measurement, and another measurement of the position may provide a different result, because the particle will no longer be found in an eigenstate concentrated at the position measured previously. Of course, the outcome of the second measurement will have been influenced by the preceding one.

a e-mail: t.m.nieuwenhuizen@uva.nl 
Instead of performing these two measurements independently in sequence, one may, in principle, also attempt to measure two non-commuting observables simultaneously. In this case, not covered by the usual formulations of the Copenhagen postulates of quantum mechanics, no erasure between the start of the measurements can take place, and indeed, all information about the particle is obtained in a single process. However, since the uncertainty principle still applies, none of it can be arbitrarily accurate; this kind of joint measurement of non-commuting observables has been studied both experimentally and theoretically [1-20].

The modified uncertainty relations for these joint measurements have been extensively studied in particular, which, among other things, led to several reformulations of the uncertainty principle, in order to more properly include the sometimes surprising uncertainties observed in these processes [1,6-15].

It has been demonstrated that, if there exists a joint measurement for all observables in a quantum correlation experiment, the experiment must satisfy the Bell inequalities and a hidden variable theory for this experiment can exist [19,20]. A similar result is known for GHZ-type experiments [20]. Finally, there exists an explicit model for a joint measurement process that includes the Hamiltonians of the measurement devices, and allows their dynamics to be treated analytically [21,22].

The present work will be primarily based on this kind of simultaneous measurement devices, and some more details will be discussed in Section 2. In Section 3 we will briefly discuss the Bell and CHSH inequalities and their relevance for joint measurements of non-commuting variables. In Section 4 the CHSH expression is evaluated analytically and its numerical values are determined. In Section 5 the results are summarized and discussed.

\section{Measuring non-commuting observables}

\subsection{A quantum measurement device with solvable dynamics}

The lack of sufficiently rich solvable models for the dynamics of quantum measurements has hampered progress in this field and in the interpretation of quantum mechanics for decades. In a series of investigations during the last decades, one of the authors, in collaboration with A. Allahverdyan and R. Balian, has put forward a set of "Curie-Weiss" models for the system and apparatus which allow analytical treatment [23,24]. It involves a Curie-Weiss magnet (CW), consisting of $N$ spin-half fermions, with Hamiltonian

$$
\hat{\mathcal{H}}=-J_{2} N \frac{\hat{m}^{2}}{2}-J_{4} N \frac{\hat{m}^{4}}{4}
$$

with parameters $J_{2}$ and $J_{4}$, chosen such that the phase transition to a magnetized state is of first order, for example: $J_{2}=0, J_{4}>0$. Here, the magnetization operator, $\hat{m}=\frac{1}{N} \sum_{n=1}^{N} \hat{\sigma}_{z}^{(n)}$ is the pointer of the apparatus. The initial state of the magnet is a metastable, paramagnetic one. At $t=0$, the apparatus is coupled to the tested spin by the interaction Hamiltonian $-g N \hat{m} \hat{s}_{z}$, and to a standard phonon bath characterized, in particular, by its temperature $T$, which lies below the first order phase transition temperature of the magnet. The interaction Hamiltonian leads to a quick decay of the so-called Schrödinger cat terms; it may be shown that $\left\langle\hat{s}_{x}\right\rangle$ and $\left\langle\hat{s}_{y}\right\rangle$ will vanish rapidly, because of a dephasing followed by decoherence [23,24]. The conditions on this type of measurement apparatuses can be relaxed [25], but in the present work we shall not elaborate on this. 
Provided the coupling parameter $g$ is large enough, the system-apparatus interaction will cause the magnet to magnetize in one of two directions: after the measurement time $\tau$ one finds $\langle\hat{m}(\tau)\rangle= \pm m_{F}(T)$ with $m_{F}(T)$ close to 1 . This indicates the magnetization of the apparatus to be either upwards or downwards in the $z$-direction. It acts as the pointer variable. At the end of this ideal measurement, the spin will be maximally correlated with it: "up" or "down" in the $z$-direction, respectively $[23,24]$.

After dephasing, decoherence and thermal relaxation, the final combined state of the apparatus $A$ and the measured spin $S$ is (for all practical purposes) described by the density matrix:

$$
\rho_{f}=p_{+} \hat{\Pi}_{m_{F}} \otimes|\uparrow\rangle\left\langle\uparrow\left|+p_{-} \hat{\Pi}_{-m_{F}} \otimes\right| \downarrow\right\rangle\langle\downarrow|,
$$

where $\hat{\Pi}_{ \pm m_{F}}$ denotes the projection operators that project onto the states with magnetizations $\pm m_{F}[25]$.

The frequencies, $p_{+}$and $p_{-}$, with which each of these two outcomes occurs in a series of measurements depends on the initial density matrix for the spin state of the ensemble of measured particles. Crucially, it has been shown that the device correctly reproduces the Born rules, and the outcome probabilities $p_{\epsilon}=\frac{1}{2}\left[1+\epsilon\left\langle\hat{\sigma}_{z}(0)\right\rangle\right]$, for magnetization directions $\epsilon= \pm 1$ along the positive or negative $z$-axis; as such, this spin-induced magnetization process may be considered to represent an ideal quantum measurement $[23,24]$.

\subsection{Measuring two variables simultaneously at one station}

This approach has also been generalized to the simultaneous measurement of two non-commuting observables [21-24,26]. Here, two Curie-Weiss magnets (CW2), are simultaneously coupled to a different component of the spin of the same particle. Again, the interaction with the particle will, under appropriate circumstances, destabilize the metastable initial state, and interaction with a reservoir drives both magnets to one of their magnetized states; dephasing followed by decoherence again causes all Schrödinger cat terms to vanish.

If both couplings are activated simultaneously, the frequency with which each combination of directions occurs again depends on the initial density matrix that describes the state of the ensemble of particles with which the magnets interact. Ideal accuracy is typically not possible in the two magnet scenario, and only occurs in the limit where the coupling between the spin and one of the two detectors is trivially set to zero, effectively reducing the system to a single ideal CW measurement. Similarly, if the two couplings are not activated simultaneously, but in sequence with a large enough time delay, the situation upon activating the first magnet becomes that of the CW measurement process, and only the spin component coupled to the first magnet is measured. The measured particle is forced into a state no longer described by its original density matrix, obscuring some of its initial properties, and preventing interactions with the second CW device from revealing anything about them, as is consistent with Heisenberg's uncertainty principle. More details may be found in the literature $[21-24,26]$. Here, we continue with both measurements starting at exactly the same moment in time.

Let us denote the station by $\mathcal{A}$ and the two apparatuses by $\mathrm{A}$ and $\mathrm{A}^{\prime}$, each having a magnet and a bath, and denote the parameters of $\mathrm{A}^{\prime}$ by a prime. As in the single magnet version, the magnetization of each magnet will become $\langle\hat{m}(\tau)\rangle= \pm m_{F}$ and $\left\langle\hat{m}^{\prime}\left(\tau^{\prime}\right)\right\rangle= \pm m_{F}^{\prime}$, respectively, indicating up or down along the corresponding axis. This will be denoted by $\epsilon= \pm 1$ for the apparatus A measuring along the $z$-axis and 
$\epsilon^{\prime}= \pm 1$ for the measurement by $\mathrm{A}^{\prime}$ along the $x$-axis. For an ensemble of particles, for which the initial expectation values of the spin components are $\left\langle\hat{\sigma}_{x}(0)\right\rangle,\left\langle\hat{\sigma}_{y}(0)\right\rangle$ and $\left\langle\hat{\sigma}_{z}(0)\right\rangle$, it may be demonstrated that the probability of each of the possible combinations of outcomes is $[21,22]$ :

$$
p_{\epsilon \epsilon^{\prime}}=\frac{1}{4}\left[1+\epsilon \lambda\left\langle\hat{\sigma}_{z}(0)\right\rangle+\epsilon^{\prime} \lambda^{\prime}\left\langle\hat{\sigma}_{x}(0)\right\rangle\right]
$$

where the absence of $\left\langle\hat{\sigma}_{y}(0)\right\rangle$ is a consequence of the "truncation of the density matrix" or "disappearance of Schrödinger cat states" observed in the CW2 setup [21-24, 26]. We recall that $\epsilon= \pm 1$ and $\epsilon^{\prime}= \pm 1$, while $\lambda$ and $\lambda^{\prime}$ are efficiency parameters $\left(0 \leq \lambda, \lambda^{\prime} \leq 1\right)$ that depend on the details of the apparatuses; numerical analysis confirms this structure, and allows one to estimate the values of $\lambda$ and $\lambda^{\prime}$ [21].

No standard "collapse of the wave function" is found: after the measurement, the particle will not be prepared in a proper eigenstate of either measured variable, nor a combination of the two, but in a more complicated state [21]. On grounds of symmetry and linearity, it can be argued that different measurement devices with the capacity to provide partial information about two different spin components should display the behavior of equation (3) [21].

Although no description as a standard measurement is possible, the process can be described as a generalized quantum measurement, corresponding to the bivariate $\operatorname{POVM}\left\{F_{\epsilon \epsilon^{\prime}}\right\}=\left\{F_{++}, F_{+-}, F_{-+}, F_{--}\right\}$, with:

$$
F_{\epsilon \epsilon^{\prime}}=\frac{1}{4}\left(\hat{\sigma}_{0}+\epsilon \lambda \hat{\sigma}_{z}+\epsilon^{\prime} \lambda^{\prime} \hat{\sigma}_{x}\right)
$$

for which it can be verified that, for any state $\rho$, one may construct the same probabilities as in (3) by computing $p_{\epsilon \epsilon^{\prime}}=\operatorname{tr}\left[\rho F_{\epsilon \epsilon^{\prime}}\right][4,21]$. For the measurements of the individual spin components, one may define $F_{\epsilon^{\prime}}^{x}=\sum_{\epsilon} F_{\epsilon \epsilon^{\prime}}$ and $F_{\epsilon}^{z}=\sum_{\epsilon^{\prime}} F_{\epsilon \epsilon^{\prime}}$.

Equivalently, one may interpret the device to simultaneously measure smeared versions of more conventional spin observables; the above may also be written as:

$$
F_{\epsilon \epsilon^{\prime}}=\frac{1}{4}\left(1-\lambda-\lambda^{\prime}\right) \hat{\sigma}_{0}+\lambda \frac{1}{4}\left(\hat{\sigma}_{0}+\epsilon \hat{\sigma}_{z}\right)+\lambda^{\prime} \frac{1}{4}\left(\hat{\sigma}_{0}+\epsilon^{\prime} \hat{\sigma}_{x}\right)
$$

where one may obtain the single-observable POVMs as before,

$$
F_{\epsilon}^{z}=(1-\lambda) \frac{1}{2} \hat{\sigma}_{0}+\lambda \frac{1}{2}\left(\hat{\sigma}_{0}+\epsilon \hat{\sigma}_{z}\right), \quad F_{\epsilon^{\prime}}^{x}=\left(1-\lambda^{\prime}\right) \frac{1}{2} \hat{\sigma}_{0}+\lambda^{\prime} \frac{1}{2}\left(\hat{\sigma}_{0}+\epsilon^{\prime} \hat{\sigma}_{x}\right)
$$

Again note that this is only possible because of the relatively small value of $\lambda$ and $\lambda^{\prime}$ : even non-commuting observables can be jointly measured if they are sufficiently smeared or diluted to become compatible, the optimal smearing value being $\lambda=1 / \sqrt{2}[4,16,27,28]$. Beyond this bound, $\left\{F_{++}, F_{+-}, F_{-+}, F_{--}\right\}$no longer defines a POVM, because some elements are no longer non-negative operators. In that case joint measurements are no longer possible. Nevertheless, both $\left\{F_{+}^{z}, F_{-}^{z}\right\}$ and $\left\{F_{+}^{x}, F_{-}^{x}\right\}$ still represent measurements that can be conducted separately with a single-apparatus setup.

For all devices that do indeed give rise to a probability distribution as in (3), one must at least remark that one requires that $\lambda^{2}+\lambda^{\prime 2} \leq 1$; otherwise, the probability would become negative for a state with $\left\langle\hat{\sigma}_{z}\right\rangle=\lambda / \sqrt{\lambda^{2}+\lambda^{\prime 2}}$ and $\left\langle\hat{\sigma}_{x}\right\rangle=\lambda^{\prime} / \sqrt{\lambda^{2}+\lambda^{\prime 2}}$; this coincides with the optimal values of the smearing parameters $[22,27,28]$. One may also define a suitable bivariate POVM with optimal values and attribute any discrepancies to the device, which acts on the measured state as a noisy channel [21]. 
For the CW2 model, the limiting value will appear to be significantly smaller; for most choices of parameters, one finds $\lambda \sim 0.3$, and no significantly larger values seem achievable in this type of device [21]. The possibility that a model exists for a device which measures two non-commuting spin components simultaneously, but does so more accurately, remains unclear.

Although neither measurement is fully accurate - even particles prepared with their spin along the positive $z$-axis will sometimes cause the detector measuring this spin component to indicate spin down - the procedure is fully informative about both spin components: as long as $\lambda$ and $\lambda^{\prime}$ are known, one may determine $\left\langle\hat{\sigma}_{x}(0)\right\rangle$ and $\left\langle\hat{\sigma}_{z}(0)\right\rangle$ for any ensemble of measured particles through repeated measurements $[21,22,26]$.

\subsection{Simultaneously measuring two variables of an entangled pair at each station}

Now, one may introduce a second copy of the two-magnet device discussed above, resulting in a total of four $\mathrm{CW}$ magnets: $\mathrm{A}$ and $\mathrm{A}^{\prime}$ at station $\mathcal{A}$, and $\mathrm{B}$ and $\mathrm{B}^{\prime}$ at station $\mathcal{B}$. As usual for EPR setups, there may be a time delay between between the simultaneous measurement at station $\mathcal{A}$ and the one at station $\mathcal{B}$. This setup will allow one to consider correlations between measurement outcomes of experiments with pairs of entangled particles. Since both devices individually should produce the result found in equation (3), one may construct the combined probability distribution for two CW2 devices. Each of them attempts to measure one of two particles from the same, possibly entangled state, so as to yield:

$$
\begin{aligned}
p_{\epsilon \epsilon^{\prime} ; \delta \delta^{\prime}}= & \frac{1}{16}\left[1+\epsilon \lambda\left\langle\left(\hat{\sigma}_{z} \otimes \hat{\tau}_{0}\right)(0)\right\rangle+\epsilon^{\prime} \lambda^{\prime}\left\langle\left(\hat{\sigma}_{x} \otimes \hat{\tau}_{0}\right)(0)\right\rangle\right. \\
& +\delta \kappa\left\langle\left(\hat{\sigma}_{0} \otimes \hat{\tau}_{z}\right)(0)\right\rangle+\delta^{\prime} \kappa^{\prime}\left\langle\left(\hat{\sigma}_{0} \otimes \hat{\tau}_{x}\right)(0)\right\rangle \\
& +\epsilon \delta \lambda \kappa\left\langle\left(\hat{\sigma}_{z} \otimes \hat{\tau}_{z}\right)(0)\right\rangle+\epsilon \delta^{\prime} \lambda \kappa^{\prime}\left\langle\left(\hat{\sigma}_{z} \otimes \hat{\tau}_{x}\right)(0)\right\rangle \\
& \left.+\epsilon^{\prime} \delta \lambda^{\prime} \kappa\left\langle\left(\hat{\sigma}_{x} \otimes \hat{\tau}_{z}\right)(0)\right\rangle+\epsilon^{\prime} \delta^{\prime} \lambda^{\prime} \kappa^{\prime}\left\langle\left(\hat{\sigma}_{x} \otimes \hat{\tau}_{x}\right)(0)\right\rangle\right] .
\end{aligned}
$$

Here $\lambda, \lambda^{\prime}, \epsilon$ and $\epsilon^{\prime}$ relate to the apparatuses $\mathrm{A}$ and $\mathrm{A}^{\prime}$ which measure $\hat{\sigma}_{z}$ and $\hat{\sigma}_{x}$ at station $\mathcal{A}$, respectively, and $\kappa, \kappa^{\prime}, \delta$ and $\delta$ 's refer similarly to the apparatuses $B$ and $B^{\prime}$ which measure $\hat{\tau}_{z}$ and $\hat{\tau}_{x}$ at station $\mathcal{B}$, respectively. Moreover, the operators $\hat{\sigma}_{0}$ and $\hat{\tau}_{0}$ denote one half times the $2 \times 2$ unit matrix, acting on the first and second particle, respectively. Again, the maximal (symmetric) values are in principle: $\lambda=\lambda^{\prime}=\kappa=\kappa^{\prime}=\frac{1}{\sqrt{2}}$, while, as mentioned earlier, the CW2 model seems to be limited to $\lambda \sim 0.29-0.30$, which easily satisfies the bounds set by both Heisenberg's principle and unitarity [21].

Devices based on a different physical principle, having an outcome probability distribution with the same form as that in (3) will, when employed at each of the stations to measure entangled states, will also be subject to (7), with their own values of $\lambda, \lambda^{\prime}, \kappa$ and $\kappa^{\prime}$.

\section{The $\mathrm{CHSH}$ inequality}

Consider a detector that, given a detector setting $a$, may measure some property, of a system in an unknown state, and may subsequently indicate a value $A(a)$. In a typical classical probabilistic theory, the expectation value of the output of this 
detector may be computed from:

$$
\langle A(a)\rangle=\int A(a, \lambda) p(\lambda) d \lambda
$$

Here $\lambda$ (not to be confused with the earlier efficiency factor) indicates some (possibly hidden) information, for which $p(\lambda)$ is the probability distribution. The integral must be taken over all values of $\lambda$. This may include properties of both the detector, and the measured system, although it is usually assumed not to depend on the choice of detector settings; see e.g. $[29,30]$ for criticism on that assumption.

Naturally, a similar formula would hold for another detector setting $a^{\prime}$ at station $\mathcal{A}$, or for another but similar detector that may be prepared in settings $b$ or $b^{\prime}$, and measures $B$ at station $\mathcal{B}$. Because of the principle of locality, it is generally assumed that the measurement outcomes do not depend on the settings of the other detector; the correlation between the outputs of two such detectors is therefore typically taken to be of the following form [31]:

$$
E(a, b)=\int A(a, \lambda) B(b, \lambda) p(\lambda) d \lambda
$$

For two-setting devices, one may then compute the following object:

$$
S_{\mathrm{CHSH}}=E(a, b)+E\left(a^{\prime}, b\right)-E\left(a, b^{\prime}\right)+E\left(a^{\prime}, b^{\prime}\right) .
$$

As long as $A$ and $B$ remain between -1 and 1 , or are normalized to do so, the so-called Clauser-Horn-Shimony-Holt (CHSH) inequality [32],

$$
\left|S_{\mathrm{CHSH}}\right| \leq 2 \text {, }
$$

must hold; a result also known as Bell's theorem [33]. In quantum theory, however, values up to $2 \sqrt{2}$ may be obtained; this result, which has been experimentally confirmed, is known as Bell's paradox [32-38].

This is by far the most well-known example of a contradiction between quantum mechanics, and local realist theories that require the aforementioned classical probabilistic structure, despite having given rise to more alternative interpretations than other such demonstrations. Unlike, for example, GHZ-type experiments, where contradictions between classical and quantum probability theory are already observed in a single run of the experiment, the $\mathrm{CHSH}-\mathrm{Bell}$ procedure requires the comparison of experiments conducted in different, incompatible contexts, which is at the root of a class of objections for which one of us has introduced the term "Contextuality loophole"; several closely related ideas have been treated in the literature $[29,30,39-51]$. The validity of these objections has, however, also been disputed [52-54]; for a reply, see [55]. Finally, it may be remarked that some objections against GHZ-type experiments have also been reported, and that certain local realist approaches, such as superdeterminism, can not be ruled out [23,39,56-59].

At each of the stations, the measurements considered in the present work, however, do take place in a single context, and in a single run of the experiment, and there exists a joint, classical probability space, that contains all information for the measurement of each variable at each of the stations $\mathcal{A}$ and $\mathcal{B}$. It will be shown that, in this case, the Bell inequalities hold.

Although without consequences for local realism, these results are connected with the close interplay between commeasurability, the possibility of measuring all pairs and triples of commuting and non-commuting observables involved, smearing and the maximal values that can be obtained for $\left|S_{\mathrm{CHSH}}\right|[16,19,20,27,28]$. 


\section{$4 \mathrm{CHSH}$ experiments in a single context at each station}

\subsection{Singlet Bell pairs}

In order to consider the CHSH inequalities, we will be interested in the interactions with fully entangled spin-zero pairs, which are to be prepared in the singlet state:

$$
\rho=\frac{1}{4}\left(\hat{\sigma}_{0} \otimes \tilde{\tau}_{0}-\sum_{i=1}^{3} \hat{\sigma}_{i} \otimes \tilde{\tau}_{i}\right) .
$$

Here, the $\hat{\sigma}_{i}$ denote the usual Pauli-matrix spin operators, applied to the first particle of an entangled pair at station $\mathcal{A}, i$ running over $(x, y, z)$, while $\tilde{\tau}_{i}$ does the same for the spin of the other particle at station $\mathcal{B}$, in the same basis.

To study correlations between non-orthogonal measurement bases, where the most interesting values of $S_{\mathrm{CHSH}}$ are found, one rotates one detector along the $y$-axis, and substitutes:

$$
\tilde{\tau}_{x}=\cos \theta \hat{\tau}_{x}+\sin \theta \hat{\tau}_{z}, \quad \tilde{\tau}_{z}=\cos \theta \hat{\tau}_{z}-\sin \theta \hat{\tau}_{x}, \quad \tilde{\tau}_{0}=\hat{\tau}_{0}, \quad \tilde{\tau}_{y}=\hat{\tau}_{y}
$$

with which one may re-express the so-called Bell (or EPR [35]) state (12) as:

$$
\begin{aligned}
\rho= & \frac{1}{4}\left[\hat{\sigma}_{0} \otimes \hat{\tau}_{0}-\hat{\sigma}_{y} \otimes \hat{\tau}_{y}-\cos \theta\left(\hat{\sigma}_{x} \otimes \hat{\tau}_{x}+\hat{\sigma}_{z} \otimes \hat{\tau}_{z}\right)\right. \\
& \left.-\sin \theta\left(\hat{\sigma}_{x} \otimes \hat{\tau}_{z}-\hat{\sigma}_{z} \otimes \hat{\tau}_{x}\right)\right]
\end{aligned}
$$

from which we can read off:

$$
\begin{aligned}
& \left\langle\hat{\sigma}_{x} \otimes \hat{\tau}_{x}\right\rangle=\left\langle\hat{\sigma}_{z} \otimes \hat{\tau}_{z}\right\rangle=-\cos \theta \\
& \left\langle\hat{\sigma}_{x} \otimes \hat{\tau}_{z}\right\rangle=-\left\langle\hat{\sigma}_{z} \otimes \hat{\tau}_{x}\right\rangle=-\sin \theta \\
& \left\langle\hat{\sigma}_{i} \otimes \hat{\tau}_{y}\right\rangle=\left\langle\hat{\sigma}_{y} \otimes \hat{\tau}_{i}\right\rangle=-\delta_{i y} \\
& \left\langle\hat{\sigma}_{0} \otimes \hat{\tau}_{i}\right\rangle=\left\langle\hat{\sigma}_{i} \otimes \hat{\tau}_{0}\right\rangle=0 .
\end{aligned}
$$

For the interaction of a pair of particles in such a state with these detectors, we may use (7) and (15) to find:

$$
p_{\epsilon \epsilon^{\prime} ; \delta \delta^{\prime}}=\frac{1}{16}\left[1-\left(\epsilon \delta \lambda \kappa+\epsilon^{\prime} \delta^{\prime} \lambda^{\prime} \kappa^{\prime}\right) \cos \theta-\left(\epsilon^{\prime} \delta \lambda^{\prime} \kappa-\epsilon \delta^{\prime} \lambda \kappa^{\prime}\right) \sin \theta\right] .
$$

Again, $\epsilon= \pm 1, \epsilon^{\prime}= \pm 1, \delta= \pm 1$ and $\delta^{\prime}= \pm 1$ denote the polarization directions of the magnets after the measurement has taken place, and $\lambda, \lambda^{\prime}, \kappa$ and $\kappa^{\prime}$ the efficiency parameters, as in (3) and (7). Note that the opposite sign in the final term stems from that in $\left\langle\hat{\sigma}_{z} \otimes \hat{\tau}_{x}\right\rangle$.

\subsection{Detector settings in a single context at each station}

To allow comparison with the conventional EPR experiment, $S_{\mathrm{CHSH}-\mathrm{sim}}$, the equivalent of $S_{\mathrm{CHSH}}$ for simultaneous measurements, is to be computed. To connect the two previous notations, we denote by $a$ the $z$-axis of Alice and by $a^{\prime}$ her $x$-axis, and by $b$ Bob's $z$-axis and by $b^{\prime}$ his $x$-axis. Note that their measurement bases have been rotated with a relative angle $\theta$. For each of the four $a-b$ combinations, we can compute the correlation, $E(a, b)$. Although both apparatuses at $A$ act simultaneously, as 
do those at $B$, one may still compare the outputs of pairs of apparatuses with one member at each station.

As in equation (9), one may compute, for example, $E(z, z)$, the correlation between the outputs of the two apparatuses corresponding to their measurements of $\hat{\sigma}_{z}$ and $\hat{\tau}_{z}$. It reads:

$$
\begin{aligned}
E(z, z) & =\sum_{\epsilon \epsilon^{\prime} \delta \delta^{\prime}= \pm 1} \epsilon \delta p_{\epsilon \epsilon^{\prime} ; \delta \delta^{\prime}}=\sum_{\epsilon^{\prime} \delta^{\prime}}\left(p_{+, \epsilon^{\prime} ;+, \delta^{\prime}}-p_{+, \epsilon^{\prime} ;-, \delta^{\prime}}-p_{-, \epsilon^{\prime} ;+, \delta^{\prime}}+p_{-, \epsilon^{\prime} ;-, \delta^{\prime}}\right) \\
& =-\lambda \kappa \cos \theta
\end{aligned}
$$

Likewise, one finds:

$$
\begin{aligned}
& E(x, z)=\sum_{\epsilon \epsilon^{\prime} \delta \delta^{\prime}} \epsilon^{\prime} \delta p_{\epsilon \epsilon^{\prime} ; \delta \delta^{\prime}}=-\lambda^{\prime} \kappa \sin \theta \\
& E(z, x)=\sum_{\epsilon \epsilon^{\prime} \delta \delta^{\prime}} \epsilon \delta^{\prime} p_{\epsilon \epsilon^{\prime} ; \delta \delta^{\prime}}=+\lambda \kappa^{\prime} \sin \theta \\
& E(x, x)=\sum_{\epsilon \epsilon^{\prime} \delta \delta^{\prime}} \epsilon^{\prime} \delta^{\prime} p_{\epsilon \epsilon^{\prime} ; \delta \delta^{\prime}}=-\lambda^{\prime} \kappa^{\prime} \cos \theta .
\end{aligned}
$$

From (7), (16), (17) and (18), one may compute the equivalent of expression (10) for simultaneous measurements. In the present notation, one finds:

$$
\begin{aligned}
S_{\mathrm{CHSH}-\operatorname{sim}} & =E(z, z)+E(x, z)-E(z, x)+E(x, x) \\
& =\sum_{\epsilon \epsilon^{\prime} \delta \delta^{\prime}= \pm 1}\left[\left(\epsilon+\epsilon^{\prime}\right) \delta+\left(-\epsilon+\epsilon^{\prime}\right) \delta^{\prime}\right] p_{\epsilon \epsilon^{\prime} ; \delta \delta^{\prime}} \\
& =-\left(\lambda \kappa+\lambda^{\prime} \kappa^{\prime}\right) \cos \theta-\left(\lambda^{\prime} \kappa+\lambda \kappa^{\prime}\right) \sin \theta .
\end{aligned}
$$

Note that, in the last step, it has been used that $\sum_{\delta} \delta=0$, while $\sum_{\delta} \delta^{2}=2$, and likewise for $\delta^{\prime}, \epsilon$ and $\epsilon^{\prime}$. Also notice the familiar combination in the middle line, where always one of the terms in round brackets vanishes. Even for (symmetric) parameters satisfying the unitarity bound, $\kappa=\kappa^{\prime}=\lambda=\lambda^{\prime}=\frac{1}{2} \sqrt{2}$, one finds $\left|S_{\mathrm{CHSH}}\right| \leq \sqrt{2}$, which fails to exceed the classically permitted value of 2 . Quantum mechanics constrains these correlations further than classical mechanics does, while, with separate measurements of each variable, it was the classical theory that was more constrained. This effect is essentially because of the unavoidable inaccuracy of the detector setup. A conventional EPR experiment with an equally limited device would not violate the Bell inequality either; but unlike such a separate measurement scenario, the detector efficiency is always limited for simultaneous measurements, because of the mutual influence the two apparatuses at each station have on each other via their couplings to the spin of the same particle.

On top of the effect that the two conflicting measurements are fundamentally required to have on each other, the CW2 model seems to suffer from an even larger reduction in efficiency. Numerical simulations of the dynamics of the CW2 detector presented in earlier work found no values $\lambda>0.28$ [21]. Our subsequent numerical analysis of the equations presented there fails to find any values $\lambda>0.3$, at least for symmetric detectors with $\lambda=\lambda^{\prime}$; typical choices of parameters result in $\lambda \sim 0.29$. For apparently optimal values, one finds $S_{\mathrm{CHSH}-\mathrm{sim}} \sim 0.24$, well below the Bell bound of $S_{\mathrm{CHSH}}=2$ and also below the simultaneous-measurement bound $S_{\mathrm{CHSH}-\mathrm{sim}}=\sqrt{2}$. 


\subsection{Different interpretation of the measurements}

It may be noted that the information provided by a measurement made with the type of device considered can also be interpreted as (a smeared version of) a measurement of a single composite variable: the spin component along one of two possible orthogonal linear combinations of the two measured spin components, $\hat{\sigma}_{u}=\frac{1}{\sqrt{\lambda^{2}+\lambda^{\prime 2}}}\left(\lambda^{\prime} \hat{\sigma}_{x}+\lambda \hat{\sigma}_{z}\right)$ and $\hat{\sigma}_{v}=\frac{1}{\sqrt{\lambda^{2}+\lambda^{\prime 2}}}\left(\lambda^{\prime} \hat{\sigma}_{x}-\lambda \hat{\sigma}_{z}\right)$, with the combination of the two magnetization directions indicating the initial spin along only one of these two axes. The device then measures along either basis with probability $\frac{1}{2}$. For this procedure, the relevant POVMs become $\left\{2 F_{++}, 2 F_{--}\right\}$for the $\hat{u}$-direction, and $\left\{2 F_{-+}, 2 F_{+-}\right\}$for the $\hat{v}$-direction; their elements being

$$
F_{\epsilon}^{u}=2 F_{\epsilon \epsilon}=\frac{1}{2}\left(\hat{\sigma}_{0}+\epsilon \tilde{\lambda} \hat{\sigma}_{u}\right), \quad F_{\epsilon}^{v}=2 F_{-\epsilon, \epsilon}=\frac{1}{2}\left(\hat{\sigma}_{0}+\epsilon \tilde{\lambda} \hat{\sigma}_{v}\right)
$$

with smearing parameter $\tilde{\lambda}=\sqrt{\lambda^{2}+\lambda^{\prime 2}}$.

It might be worth noting that more smearing is required to render two noncommuting variables commeasurable than to satisfy the Bell inequalities: saturation occurs when the smearing parameter becomes $\tilde{\lambda}=2^{-\frac{1}{4}}$, while for simultaneous measurement of $\left\{F_{\epsilon}^{u}\right\}$ and $\left\{F_{\epsilon}^{v}\right\}$ to be possible, one requires $\tilde{\lambda} \leq 2^{-\frac{1}{2}}[27]$. If this is not the case, some elements of the corresponding bivariate POVM fail to be non-negative operators. In any model for which the probabilities of outcomes can be coded as in (3), Bell saturation occurs for the composite variables when $\lambda=\lambda^{\prime}=2^{-\frac{3}{4}} \sim 0.59$; twice the maximal value found in the CW2 model.

\section{Summary and discussion}

The Curie-Weiss model for ideal measurements of the $z$-component of a spin- $\frac{1}{2}$ fermion is known to be exactly solvable [23] and can been extended to incorporate the simultaneous measurement of the $z$ and $x$-components of a similar spin- $\frac{1}{2}$ state, by adding a second CW device, coupled to the second observable [21-23]. In an EPR setup, there are two stations where such a simultaneous measurement takes place, with a possible but irrelevant time delay between them. The correlations between the outcomes of measurements on pairs of entangled particles, are presented.

Our numerical analysis of the analytical solution of an explicit example of such coupled devices, and results in the literature for this model, support these finds [21]. The maximal efficiency with which devices of this kind may measure either variable is strongly constrained, at least partially because the uncertainty principle and the influence the two measurement processes have on each other through their coupling to the measured particle.

In the type of experiment considered, measurements of each observable involved take place in every individual run of the experiment, and in a single context. Hence there must exist a single probability distribution that represents the frequency with which each outcome occurs; in this type of scenario, the Bell inequalities hold, which is consistent with the analysis in the present and previous work $[19,21,22,40,44]$.

A stronger bound than that of Bell $\left(\left|S_{\mathrm{CHSH}-\mathrm{sim}}\right| \leq \sqrt{2}\right.$ instead of $\left.\left|S_{\mathrm{CHSH}}\right| \leq 2\right)$ is shown to hold for a class of joint measurement processes that probe two noncommuting variables with equal susceptibility, which confirms earlier results on such measurements $[27,28]$. Here, we consider Curie-Weiss-type detectors and take into account the internal dynamics of the measurement devices for an example of such a 
process, using methods presented in earlier work [21]. The values of $\left|S_{\mathrm{CHSH}-\mathrm{sim}}\right|$ are actually found to lie well below $\sqrt{2}$.

Any procedure to simultaneously measure incompatible observables may only provide limited information about each of the properties of the initial state, because the attempt to measure one variable will inevitably disturb the measurement of the other. Even an ensemble of spins prepared along a measurement axis will cause both outputs to occur with finite, although non-trivial frequency. This effect is similar to that responsible for Heisenberg's uncertainty relations [1,7,11-14]. Usually, such effects are observed in measurements performed in sequence, where a system will not be in a proper eigenstate of one observable after another observable, which does not commute with the first, has been measured. In our situation, however, measuring the two observables at the respective stations simultaneously, prevents the measured state from forming a proper eigenstate of the other during any stage of the measurement process. The final state, in particular, is an eigenstate of neither. This alone, however, is insufficient to explain the detector efficiency observed in numerical analysis of our model, which suggests that the specifics of the CW2 measurement device are at least partially responsible; it is conceivable that a device with similar capacities, but based on a different physical mechanism, is more accurate. Regardless of these complications, the procedure is informative, in the sense that it does allow one to determine expectation values of both measured observables for an ensemble of measured particles.

This research was supported (in part) by the John E. Fetzer Memorial Trust.

Open Access This is an open access article distributed under the terms of the Creative Commons Attribution License (http://creativecommons.org/licenses/by/4.0), which permits unrestricted use, distribution, and reproduction in any medium, provided the original work is properly cited.

\section{References}

1. E. Arthurs, J.K. Kelly Jr., Bell Syst. Tech. J. 44, 725 (1965)

2. W.M. de Muynck, W. De Baere, H. Martens, Found. Phys. 24, 1589 (1994)

3. W.M. de Muynck, H. Martens, Phys. Rev. A 42, 5079 (1990)

4. W.M. de Muynck, Foundations of Quantum Mechanics, an Empiricist Approach (Kluwer Academic Publishers, Dordrecht, 2002)

5. N. Gisin, arXiv:1708.05556 (2017)

6. M. Ringbauer, D.N. Biggerstaff, M.A. Broome et al., Phys. Rev. Lett. 112, 020401 (2014)

7. M.J.W. Hall, Phys. Rev. A 69, 052113 (2004)

8. H. de Raedt, K. Michielsen, Front. Phys. 2, 14 (2014)

9. J. Erhart, S. Sponar, G. Sulyok et al., Nat. Phys. 8, 185 (2012)

10. S. Sponar, G. Sulyok, J. Erhart et al., Phys. Rev. A 88, 022110 (2013)

11. M. Ozawa, Phys. Rev. Lett. 60, 385 (1988)

12. M. Ozawa, Phys. Rev. A 67, 042105 (2003)

13. M. Ozawa, J. Phys.: Conf. Ser. 504, 012024 (2014)

14. R.F. Werner, arXiv:quant-ph/0405184 (2004)

15. S. Hacohen-Gourgy, L.S. Martin, E. Flurin et al., Nature 538, 491 (2016)

16. P. Busch, Found. Phys. 17, 905 (1987)

17. G. Pütz, T. Barnea, N. Gisin, A. Martin, arXiv:1610.04464 (2016)

18. S. Stenholm, Ann. Phys. 218, 233 (1992)

19. A. Fine, Phys. Rev. Lett. 48, 291 (1982)

20. W. Son, E. Andersson, S.M. Barnett, M.S. Kim, Phys. Rev. A 72, 052116 (2005) 
21. M. Perarnau-Llobet, T.M. Nieuwenhuizen, Phys. Rev. A 95, 052129 (2017)

22. A.E. Allahverdyan, R. Balian, T.M. Nieuwenhuizen, Physica E 42, 339 (2010)

23. A.E. Allahverdyan, R. Balian, T.M. Nieuwenhuizen, Phys. Rep. 525, 1 (2013)

24. A.E. Allahverdyan, R. Balian, T.M. Nieuwenhuizen, Europhys. Lett. 61, 452 (2003)

25. A.E. Allahverdyan, R. Balian, T.M. Nieuwenhuizen, Ann. Phys. 376, 324 (2017)

26. A.E. Allahverdyan, R. Balian, T.M. Nieuwenhuizen, Phys. Rev. Lett. 92, 120402 (2004)

27. P. Busch, P. Lahti, J.-P. Pellonpää, K. Ylinen, Quantum Measurement (Springer International Publishing, Switzerland, 2016)

28. M. Banik, M.R. Gazi, S. Ghosh et al., Phys. Rev. A 87, 052125 (2013)

29. K. Hess, W. Philipp, Proc. Natl. Acad. Sci. 98, 14224 (2001)

30. K. Hess, Einstein was right! (Pan Stanford Publishing, Singapore, 2014)

31. E.T. Jaynes, Maximum Entropy and Bayesian Methods (Kluwer, Dordrecht, 1989)

32. J.F. Clauser, M.A. Horne, A. Shimony, R.A. Holt, Phys. Rev. Lett. 23, 880 (1969)

33. J.S. Bell, Physics 1, 195 (1964)

34. B.S. Cirel'son, Lett. Math. Phys. 4, 93 (1980)

35. A. Einstein, B. Podolsky, N. Rosen, Phys. Rev. 47, 777 (1935)

36. J.S. Bell, Speakable and Unspeakable in Quantum Mechanics: Collected Papers on Quantum Philosophy (Cambridge University Press, Cambridge, 2004)

37. A. Aspect, P. Grangier, G. Roger, Phys. Rev. Lett. 49, 91 (1982)

38. B. Hensen, H. Bernien, A.E. Dréau et al., Nature 526, 682 (2015)

39. D.M. Greenberger, M.A. Horne, A. Shimony, A. Zeilinger, Am. J. Phys. 58, 1131 (1990)

40. T.M. Nieuwenhuizen, Found. Phys. 41, 580 (2011)

41. J.H. Eberly, Contemp. Phys. 56, 407 (2015)

42. A. Khrennikov, Found. Phys. 32, 1159 (2002)

43. A. Khrennikov, arXiv:0709.3909 (2007)

44. A. Auffèves, P. Grangier, Found. Phys. 46, 121 (2016)

45. M. Kupczynski, Phys. Lett. 116, 417 (1986)

46. M. Kupczynski, J. Russ. Laser Res. 26, 514 (2006)

47. M. Kupczynski, Am. Inst. Phys. Conf. Ser. 861, 516 (2006)

48. F. de Zela, Sci. Rep. 7, 14570 (2017)

49. K. Hess, K. Michielsen, H. de Raedt, Europhys. Lett. 87, 60007 (2009)

50. M. Kupczynski, AIP Conf. Proc. 1327, 394 (2011)

51. H. de Raedt, K. Hess, K. Michielsen, J. Comput. Theor. Nanosci. 8, 1011 (2011)

52. C.M. Care, arXiv:1602.02628 (2016)

53. I. Schmelzer, Found. Phys. 47, 117 (2017)

54. J.P. Lambare, J. Pastor, Found. Phys. 47, 1591 (2017)

55. T.M. Nieuwenhuizen, M. Kupczynski, Found. Phys. 47, 316 (2017)

56. G. 't Hooft, arXiv:1405.1548 (2014)

57. L.E. Szabó, A. Fine, Phys. Lett. A 295, 229 (2002)

58. A. Khrennikov, Phys. Lett. A 278, 307 (2001)

59. K. Svozil, New J. Phys. 8, 39 (2006) 